\title{
ARTIGO ORIGINAL \\ CASOS DE DOENÇA DE CHAGAS AGUDA NO ESTADO DO TOCANTINS: MAPEAMENTO POR MUNICÍPIOS ENTRE 2008 E 2018
}

\author{
CASES OF ACUTE CHAGAS DISEASE IN THE STATE OF TOCANTINS: \\ MAPPING BY MUNICIPALITIES BETWEEN 2008 AND 2018
}

\author{
Viníccius Marques Fernandes Mozer', Guilherme Parreira Vaz' Evandro Leite \\ Bitencourt $^{2}$, Sabina Borges da Costa ${ }^{3}$, Arthur Alves Borges de Carvalho ${ }^{4}$.
}

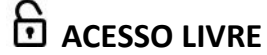

Citação: Mozer VMF, Vaz GP, Bitencourt EL, Costa SB, Carvalho AAB (2020) Casos de doença de chagas aguda no estado do Tocantins: mapeamento por municípios entre 2008 e 2018. Revista de Patologia do Tocantins, 7(1):.22-26

\section{Instituição:}

${ }^{1}$ Acadêmico Medicina, Universidade Federal do Tocantins, Palmas, Brasil. ${ }^{2}$ Graduado em Química. Mestre em Química. Acadêmico Medicina, Universidade Federal do Tocantins. Pesquisador Instituto Médico Legal do Tocantins (IML/TO), Palmas, Brasil.

${ }^{3}$ Enfermeira. Pós graduada em Gestão em Saúde da Família. Pós graduada em Enfermagem em Cardiologia. Mestra em Gestão Organizacional, Universidade Federal de Goiás, Regional Catalão. Coordenadora do SAMU Catalão/GO. ${ }^{4}$ Graduado em Medicina, Mestre em Patologia, Médico Patologista do Serviço de Verificação de Óbitos de Palmas-TO (SVO-TO), Professor do curso de Medicina da Universidade Federal do Tocantins, campus Palmas.

\section{Autor correspondente: Guilherme}

Parreira Vaz ; parreirameduft@gmail.com

Editor: Guedes V. R. Medicina, Universidade Federal do Tocantins, Brasil.

Publicado: 28 de junho de 2020.

Direitos Autorais: (c) 2020 Mozer et al. Este é um artigo de acesso aberto que permite o uso, a distribuição e a reprodução sem restrições em qualquer meio, desde que o autor original e a fonte sejam creditados.

Conflito de interesses: os autores declararam que não existem conflitos de interesses.

\begin{abstract}
RESUMO
A Doença de Chagas (DC) é a infecção causada pelo protozoário Trypanosoma cruzi. Apresenta uma fase aguda, a qual caracteriza a morbidade como Doença de Chagas Aguda (DCA). A DCA pode cursar com sinais moderados ou inexistentes, mas em sua fase crônica há o desenvolvimento da insuficiência cardíaca e problemas digestivos graves. O objetivo do artigo é demostrar e discutir o mapeamento por municípios do número de casos da Doença de Chagas Aguda (DCA), entre 2008 e 2018, no estado do Tocantins. Para isso, foi utilizado como metodologia um estudo do tipo epidemiológico, de natureza descritiva, realizado por meio da coleta o número total de casos confirmados da Doença de Chagas Aguda, disponibilizado pelo (SINAN) do Departamento de Informação e Informática do SUS (DATASUS). Foi obtido um total de 47 casos confirmados da DCA entre 2008 e 2018 no estado do Tocantins. Desses, 26 casos em pacientes do sexo feminino e 21 do sexo masculino. Esses se concentraram nas seguintes cidades: Ananás (11), Aparecida do Rio Negro (10) e Axixá do Tocantins (9) respectivamente. Além disso, observou-se o padrão oral de contaminação (37 possíveis casos) como maior forma de infecção em comparação a 5 casos por provável transmissão vetorial. Dessa forma, a transmissão oral da doença é expressiva e preocupante. Destaca-se a necessidade de investimentos para a fiscalização do comércio de açaí, bem como a elaboração e execução de um modelo consistente de investigação epidemiológica local do comportamento da Doença de Chagas Aguda.

Palavras-chave: Doença de Chagas. Epidemiologia. Saúde.
\end{abstract}

\begin{abstract}
Chagas disease (CD) is an infection caused by the protozoan Trypanosoma cruzi. It presents an acute phase, which characterizes morbidity as Acute Chagas Disease (ACD). ACD can present with moderate or nonexistent signs, but in its chronic phase there is the development of heart failure and severe digestive problems. The objective of the article is to demonstrate and discuss the mapping by municipalities of the number of cases of Acute Chagas Disease (ACD), between 2008 and 2018, in the state of Tocantins. For this purpose, an epidemiological study of a descriptive nature was used as methodology, carried out by collecting the total number of confirmed cases of Acute Chagas Disease, made available by (SINAN) of the SUS Information and Informatics Department (DATASUS). A total of 47 confirmed cases of ACD were obtained between 2008 and 2018 in the state of Tocantins. Of these, 26 cases in female and 21 male patients. These were concentrated in the following cities: Ananás (11), Aparecida do Rio Negro (10) and Axixá do Tocantins (9) respectively. In addition, the oral pattern of contamination (37 possible cases) was observed as a greater form of infection compared to 5 cases due to probable vector transmission. Thus, the oral transmission of the disease is expressive and worrisome. We highlight the need for investments for the inspection of the açaí trade, as well as the elaboration and execution of a consistent model of local epidemiological investigation of the behavior of the Acute Chagas Disease.
\end{abstract}

Keywords: Chagas Disease. Epidemiology. Health. 


\section{INTRODUÇÃO}

A doença de Chagas (DC) tem como agente etiológico o hemoprotozoário flagelado Trypanosoma cruzi, o qual circula entre insetos vetores (Triatominae, Hemiptera, Reduviidae) chamado popularmente de "barbeiros"1. O protozoário é transmitido pelo contato com as fezes dos vetores, sendo que a morbidade também pode ser transmitida por transfusão de sangue ou na gravidez, da mãe infectada ao feto ${ }^{1}$.

Sua descoberta se deu em 1909, quando o cientista brasileiro Carlos Chagas estudava a malária ${ }^{2}$. 0 médico não só detectou o causador da doença em humanos, como também descobriu o vetor descrevendo a patologia da $D C^{2}$. Desde sua evidenciação, muitos pesquisadores contribuíram para o melhor entendimento dos seus processos endêmicos, porém uma análise de controle bem elaborada envolve múltiplos aspectos e abordagens ${ }^{2}$.

Sabe-se atualmente que ao ser contraído, o patógeno pode acarretar diversos tipos de lesões e atingir órgãos vitais, como fígado, coração e musculatura esquelética ${ }^{3}$. Todavia, apesar do diagnóstico da patologia se dar em sua grande maioria por exames de sangue realizados gratuitamente pelo SUS, as suspeitas diagnósticas devem ser baseadas também em contextos de risco e vulnerabilidade, como o local de residência ou rede social do paciente, pois parte dos casos não apresenta sintomas ${ }^{3}$.

Nesse contexto, até meados de 1950, a Doença de Chagas era considerada predominantemente rural, em áreas mais próximas de reservatórios dos vetores ${ }^{4}$. Entretanto, levando-se em conta as transformações socioambientais e a urbanização que o Brasil vivencia principalmente na região Norte do país, a moléstia ganhou uma contextualização epidemiológica mais urbana ${ }^{4}$. Isso pode ser evidenciado por dados do Ministério da Saúde relativos à vigilância entomológica de 2007 a 2011, que indicam o registro da captura de mais de $\mathbf{7 7 0}$ mil triatomíneos nos contextos locais de domicílios ${ }^{4}$.

Convém lembrar ainda que a distribuição espacial da doença é limitada primariamente ao continente americano em virtude da distribuição de mais de 140 espécies do inseto vetor, a maioria na América Latina 5 . De acordo com a OMS, com base em dados de 2010, quase $1 / 5$ das mais de 100 milhões de pessoas infectadas por T. cruzi eram brasileiras ${ }^{5}$. Além disso, estimativas de 2015 apontam que cerca de $80 \%$ dos acometidos não têm acesso a diagnóstico e tratamento, o que sustenta o custo social da enfermidade ${ }^{5}$.

Por sua vez na região Norte do Brasil, a transmissão oral tem assumido importância maior, visto que somado à vetorial, também pode ser transmitida por alimentos contaminados com os vetores triturados ou os seus dejetos ${ }^{6}$. Nessa ótica, a veiculação do $T$. cruzi pela polpa de açaí tem assumido protagonismo na discussão, pois esta fruta é bastante consumida por toda a região amazônica ${ }^{6}$. Assim, observa-se que em 2006, ano em que a forma oral foi identificada como de potencial risco para a Saúde Pública, foram notificados 116 casos de Doença de Chagas Aguda em todo o Brasil ${ }^{6}$.
Nesse contexto, pode-se notar que mesmo com algumas medidas tomadas pela gestão pública e pelo Ministério da Saúde voltadas ao controle entomológico, como o uso de inseticidas de ação residual e a campanha antitriatomínica, mais de cem anos se passaram desde sua identificação e a problemática persiste ${ }^{7}$. Desta feita, uma vez que esta engloba diversos mecanismos socioculturais, políticos e econômicos, a tripanossomíase americana ainda apresenta um número de casos significativos ${ }^{7}$.

Tal fato comprova-se de acordo com estudos sobre a mortalidade decorrente da doença de Chagas entre 2010 e 2013, no qual o Brasil registrou 18.827 óbitos pela mesma ${ }^{8}$. A região Norte foi responsável por $2 \%$ das vítimas de DC no país, e o estado do Tocantins o local de ocorrência de $59 \%$ dessas mortes $^{8}$.

Isso demonstra a relevância do estudo sobre a epidemiologia local da morbidade para evidenciar a evolução de seu comportamento, útil às políticas de combate $\mathrm{e}$ prevenção ao vetor. Dessa maneira, o objetivo do presente artigo é demostrar e discutir o mapeamento por município do número de casos da Doença de Chagas Aguda (DCA), entre 2008 e 2018, no estado do Tocantins.

\section{METODOLOGIA}

Trata-se de um estudo do tipo epidemiológico, de natureza descritiva, realizado por meio da coleta de dados anuais disponibilizados pelo Sistema de Informação de Agravos de Notificação (SINAN) do Departamento de Informação e Informática do SUS (DATASUS). As informações coletadas foram o número total de casos confirmados da Doença de Chagas Aguda, referentes ao período entre 2008 e 2018, no estado do Tocantins (TO).

Esta informação estatística foi analisada segundo as variáveis: idade, sexo, município de residência, local provável de infecção e forma provável de infecção. Para isso, foram utilizadas todas as faixas etárias disponíveis entre 0 e acima de 80 anos permitindo o mapeamento detalhado da morbidade durante o período descrito. A partir dos dados obtidos foi realizada uma análise descritiva simples e os achados mais significativos apresentados em tabelas.

\section{RESULTADOS}

A partir da pesquisa epidemiológica foi obtido um total de 47 casos confirmados da Doença de Chagas Aguda entre 2008 e 2018 no estado do Tocantins. Desses, 26 casos em pacientes do sexo feminino e 21 do sexo masculino. Quanto às faixas etárias predominantes destacaram-se 20-39 anos e 40-59 anos com 17 e 12 casos confirmados respectivamente. A tabela abaixo, fornecida através do acesso ao DATASUS, representa a distribuição do número total de casos de acordo com o sexo e faixa etária ${ }^{9}$.

Tabela 1: Evolução do número de casos da Doença de Chagas Aguda segundo sexo e faixa etária no estado do Tocantins entre 2008 e 2018. 


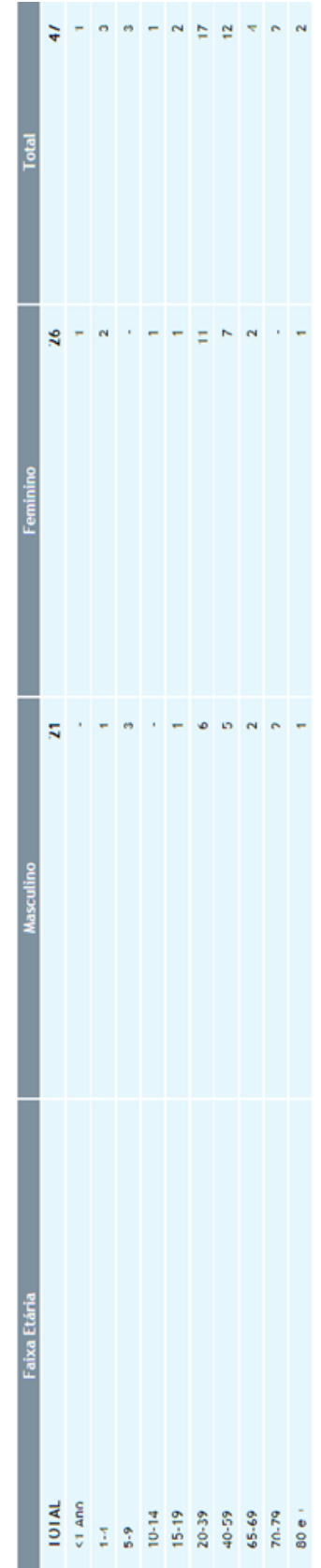

FONTE: Ministério da Saúde/SVS - Sistema de Informação de Agravos de Notificação - SINAN Net, 2020.

Por sua vez, o mapeamento da DCA por município no TO evidenciou o predomínio da incidência dessa morbidade nas seguintes cidades: Ananás (11), Aparecida do Rio Negro (10) e Axixá do Tocantins (9) respectivamente. Juntas correspondem a $63,8 \%$ dos casos registrados no período de estudo, demonstrando relevância significativa na progressão da evolução dessa doença. Nessas localidades, sua ocorrência também foi predominante no sexo feminino em pelo menos duas das três regiões com maior incidência da $D C^{9}$.

A tabela abaixo permite a análise do número total de casos da DCA registrados entre 2008 e 2018 em todos os municípios do estado do Tocantins, segundo sexo e local de residência.

Tabela 2- Evolução do número de casos da Doença de Chagas Aguda segundo município de residência e sexo no estado do Tocantins entre 2008 e 2018.

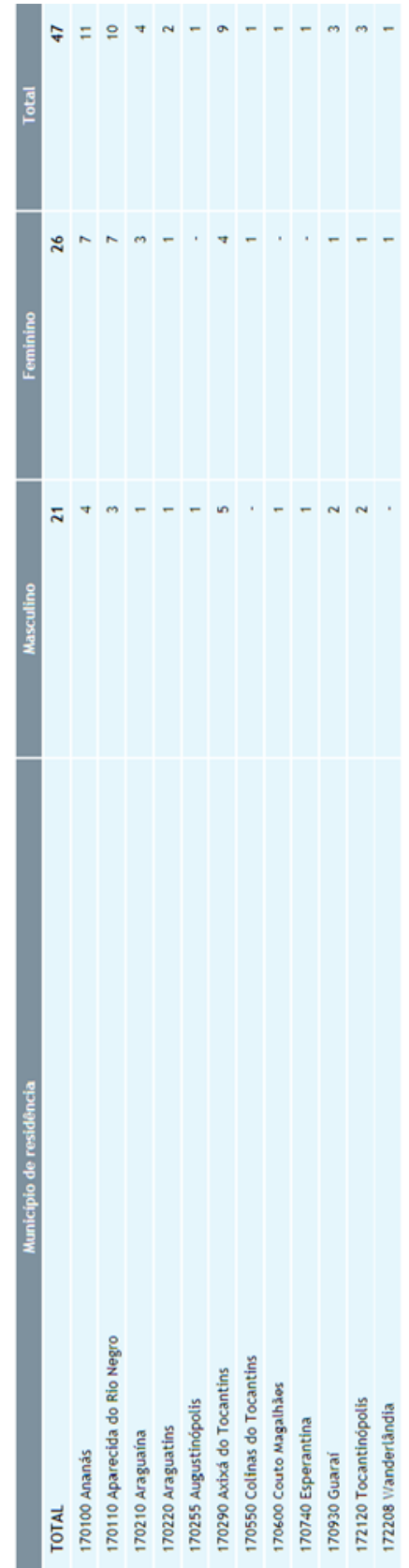

FONTE: Ministério da Saúde/SVS - Sistema de Informação de Agravos de Notificação - SINAN Net, 2020.

Quanto à análise do número de casos confirmados de DCA segundo local provável de infecção, a pesquisa mostrou o predomínio da infecção domiciliar, com 31 casos em detrimento de outras prováveis localidades. Além disso, foi possível observar o padrão oral de contaminação (37 possíveis casos) como maior forma de infecção em comparação a 5 casos por provável transmissão vetorial. A tabela abaixo representa a relação entre local provável de infecção e forma provável de infecção por DCA no estado do Tocantins dentro do período analisado?

Tabela 3- Relação de casos confirmados da Doença de Chagas Aguda segundo local provável de infecção e modo provável de infecção no estado do Tocantins entre 2008 e 2018. 


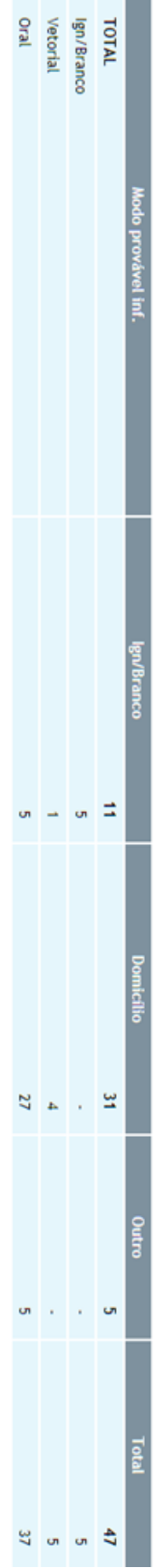

FONTE: Ministério da Saúde/SVS - Sistema de Informação de Agravos de Notificação - SINAN Net, 2020.

A região de abrangência da Amazônia Legal compreende a totalidade dos estados do Acre, Amapá Amazonas, Mato Grosso, Pará, Rondônia, Roraima, Tocantins e parte do Maranhão ${ }^{10}$. Embora o número total de casos da DCA no estado do Tocantins não seja tão representativo ao se analisar a dinâmica e comportamento da doença em torno do mesmo observa-se um fenômeno de expansão da morbidade a qual possui várias explicações a serem consideradas ${ }^{10}$.

Sabe-se que atualmente, um dos estados brasileiros que possui o maior número confirmado de casos agudos da DC é o Pará (PA) (cerca de 70\%), também pertencente à Amazônia Legal e vizinho do estado do Tocantins ${ }^{10}$. Além disso, o conhecimento da cadeia de transmissão da Doença de Chagas na região amazônica até hoje não está devidamente caracterizado e representa um grande desafio para a compreensão da magnitude da $D_{C A}{ }^{10,11}$. Exemplo disso é a falta de informação sistematizada que apoie a definição das diretrizes e tomada de decisões junto a programas de prevenção, rastreio e controle da mesma ${ }^{10,11}$.

Um aspecto interessante a ser discutido é o fato de que o gatilho para todo o processo de atenção em saúde na região amazônica, referente à $\mathrm{DC}$, ocorreu após o surto de transmissão oral desta por caldo de cana em Santa Catarina $(\mathrm{SC})^{11}$. Esse acontecimento contribuiu para mudanças de estratégias políticas e o fortalecimento da vigilância em saúde na Amazônia Legal, com enfoque primordial para a contaminação oral da Doença de Chagas ${ }^{11}$.

Todavia, um dado relevante evidenciado por essa pesquisa epidemiológica foi o predomínio de casos confirmados por provável infecção de DCA através da via oral em municípios do $\mathrm{TO}^{12}$. A via oral de transmissão dessa morbidade tem sido amplamente comprovada por diversos autores demonstrando sua importância, frequência e visibilidade na cadeia epidemiológica da $D C^{12}$.

Sabe-se que até o ano de 2004 a ocorrência da DCA por transmissão oral, relacionada ao consumo de alimentos, constituía um evento pouco conhecido e investigado ${ }^{12,13}$. No entanto, atualmente esses surtos tornaram-se frequentes na região amazônica e em diversos estados brasileiros. Os casos recentes de DCA notificados no Brasil estão relacionados ao consumo de suco de açaí, alimento essencial na dieta da população da Região Norte ${ }^{12,13}$.

Nesse contexto, tal realidade pode ser justificada inicialmente pelo fato de o açaí ser o bem de consumo diário de muitas pessoas das localidades em questão, inclusive pelo preço acessível e elevado valor nutricional ${ }^{13}$. Associado a isso, o ecoturismo tem se desenvolvido significativamente nos estados do norte brasileiro, atraindo pessoas de diversas partes do país e até mesmo de outros países, as quais consomem da autêntica culinária regional ${ }^{13}$.

Consequentemente, os turistas são expostos ao risco de contaminação podendo ser infectados e contraírem a $\mathrm{DCA}^{13,14}$. Outro aspecto a ser considerado refere-se a pouca efetividade do congelamento do açaí como método do controle da transmissão, uma vez que o parasito demonstrou uma capacidade de adaptação formidável frente ao estresse térmico combinado a 4 o graus de temperatura ambiente ${ }^{13,14}$. Isso aponta uma problemática ainda mais grave, que é a possibilidade de transmissão da doença entre países através da exportação do açaí brasileiro ${ }^{13,14}$.

Por sua vez, vários problemas que norteiam a qualidade sanitária dos alimentos produzidos a partir desse fruto têm levado os sistemas públicos de saúde ao enfrentamento de uma nova demanda que é o combate à DCA por consumo de açaí ${ }^{14}$. A escassez de dados com relação a esse tipo de transmissão aliada à falta de programas de capacitação dos manipuladores, de tecnologia para o processamento do fruto e do controle de sua qualidade tornam o seu consumo um sério risco à saúde do consumidor $^{14}$.

Nessa perspectiva, algumas ações realizadas em âmbito governamental merecem discussão no intuito de se expor melhor a importância do aprimoramento de pesquisas e políticas públicas de saúde voltadas para a $D A^{14,15}$. No caso particular do açaí, o Ministério da Agricultura, Pecuária e Abastecimento, através da EMBRAPA (Empresa Brasileira de Pesquisa Agropecuária), elaborou um conjunto de 
procedimentos necessários para o seu processamento, incluindo etapas desde a colheita do alimento até o estoque do mesmo ${ }^{14,15}$.

No entanto, a falta de fiscalização efetiva das indústrias e demais segmentos da cadeia produtiva facilitam a ocorrência de inadequações as quais culminam na contaminação da população pela morbidade ${ }^{15}$. Já em 2009, o Ministério da Saúde elaborou o "Guia de vigilância, prevenção, controle e manejo clínico da doença de chagas aguda transmitida por alimentos". Essa ferramenta se mostrou bastante útil para o planejamento e monitoramento da DCA feito pelas redes de saúde ${ }^{15}$.

Por outro lado, a falta de capacitações e incentivo à Atenção Básica em Saúde, a qual realiza o acompanhamento diário da população dos municípios é um agravante que dificulta o controle do número de casos confirmados ${ }^{15}$, 16 . Isso é evidenciado pelo número crescente de subnotificações, motivo esse, talvez, uma das possíveis causas do número relativamente baixo de notificações da DCA nos municípios do Tocantins ${ }^{15,16}$.

Por fim, a Agência Nacional de Vigilância Sanitária (ANVISA) realizou a criação de um informe técnico sobre o gerenciamento do risco sanitário na transmissão da DCA por alimentos e ressaltou a importância da pesquisa científica nas seguintes temáticas: viabilidade, técnicas de detecção e de inativação e formas de contaminação de $T$. cruzi nos alimentos, bem como o desenvolvimento de medidas de controle consistentes ${ }^{16}$. Tal fato reafirma o quão dinâmico e relevante é a Doença de Chagas, realidade a qual representa um novo desafio à saúde pública ${ }^{16}$.

\section{CONCLUSÃO}

O estudo epidemiológico aponta o crescimento significativo do número de casos da Doença de Chagas Aguda nos municípios do estado do Tocantins durante o período analisado. Além disso, foi possível considerar a transmissão oral da doença como expressiva e preocupante, uma vez que existem grandes desafios para o controle das vias de contaminação pela mesma. Dentre eles destaca-se a necessidade de investimentos para a fiscalização do comércio de açaí e mecanismos legais para a punição frente ao descumprimento das normas pelas indústrias, bem como a elaboração e execução de um modelo consistente de investigação epidemiológica local do comportamento da DCA nos municípios do país.

\section{REFERÊNCIAS BIBLIOGRÁFICAS}

1. Kropf SP et al. Doença de Chagas: a construção de um fato científico e de um problema de saúde pública no Brasil. Revista Ciência \& Saúde Coletiva. Vol. 5(2): 347-365, 2000

2. Sangenis LH et al. Transmissão da Doença de Chagas por consumo de carne de caça: revisão sistemática. Rev. bras. epidemiol. 19 (04) Oct-Dec 2016.

3. Westphalen EV et al. Aspectos Epidemiológicos e históricos do controle da doença de Chagas no Continente Americano. BEPA, Bol. epidemiol. paul. (Online) vol.9 no.105 São Paulo set. 2012

4. Monteiro AC et al. Doença de Chagas: uma enfermidade descoberta por um brasileiro. Saúde em Foco, Edição no: 07/Ano: 2015.
5. Ministério da Saúde. Superintendência de Campanhas de Saúde Pública. Doença de Chagas: textos de apoio. Brasília: Ministério da Saúde. Sucam, 1989.

6. Araújo SM, Castilho JM. Doença de Chagas. News: artigos Cetrus. 2014.

7. Manuseio Clínico da Doença de Chagas na Fase Aguda: o desafio continua no século 21. Arq Bras Cardiol. 2019; Vol.112(3): 247-248.

8. Kashiwabara YB et al. Doença de Chagas: revisão de literatura. Braz. J. Surg. Clin. Res. V.4, n.3, pp.49-52 (Set Nov. 2013)

9. Ministério da Saúde. DATASUS. http://datasus.saude.gov.br/informacoes-de-saude-tabnet/ Acesso em 12/04/2020.

10. Santos SO. Eco- epidemiologia da Doença de Chagas Aguda em área amazônica: município de Abaetetura, estado do Pará, Brasil 2008-2009. Dissertação: Doutorado em Ciências Biológicas. Programa de Pós Graduação em Ciências Biológicas. Universidade Federal de Ouro Preto, 2013.

11. Ribeiro AS et al. Análise dos aspectos epidemiológicos e históricos do controle da Doença de Chagas. Revista Temas em Saúde. Vol17(1), 2017.

12. Dias JC et al. Doença de Chagas na Amazônia: esboço da situação atual e perspecti-vas de prevenção. Revista da Sociedade Brasileira de Medicina Tropical. Vol.35(6): 669678 nov-dez, 2002.

13. Figueira $C B$ et al. Reincidência da Doença de Chagas no Brasil por vias alternativas de transmissão: revisão sistemática. Revista de Patologia do Tocantins 2019; 6(2): 61-64.

14. Brasil. Ministério da Saúde. Doença de Chagas Aguda: aspectos epidemiológicos, diagnóstico e tratamento. Guia de consulta para profissionais da saúde. Brasília, DF, 2009.

15. Ferreira RT et al. Transmissão oral da Doença de Chagas pelo consumo de açaí: um desafio para a vigilância sanitária. Revista Vigilância Sanitária Debate 2014; 2(04): 411.

16. Vargas A et al. Investigação de surto de Doença de Chagas Aguda na região extra-amazônica, Rio Grande do Norte, Brasil, 2016. Revista Cad. Saúde Pública. Vol. 34(1), 2018. 